\title{
ORIGINAL
}

\section{EFECTO SOBRE EL CONSUMO DE RECURSOS HOSPITALARIOS DE UN PROGRAMA DE ATENCIÓN GERIÁTRICA DOMICILIARIA EN PERSONAS ANCIANAS CON PATOLOGÍA CARDIORRESPIRATORIA MUY EVOLUCIONADA}

\author{
Alejandro Pérez Martín, M. ${ }^{a}$ José Rol de la Morena, Manuel Mareque Ortega, Mercedes Gómez \\ Gómez, Carmen Gómez Gómez y Matilde Díaz de Cerio.
}

Unidad de Atención Domiciliaria Hospital Geriátrico Virgen del Valle. Toledo.

\section{RESUMEN}

Fundamento: Los equipos de atención geriátrica domiciliaria tienen entre sus objetivos el seguimiento de pacientes ancianos con alto riesgo de reingreso hospitalario. Aunque llevan años funcionando en nuestro país, no se tienen datos sobre la repercusión que dicho seguimiento representa en el consumo de recursos hospitalarios. El objetivo del presente estudio es analizar el efecto que supone, sobre dichos recursos, el control por una unidad de atención geriátrica domiciliaria de pacientes con patología cardiorrespiratoria crónica muy evolucionada.

Métodos: En el estudio se incluyó a los pacientes con patología cardiorrespiratoria crónica atendidos por la unidad entre enero de 1995 y enero de 1999, habiéndose excluido a los que permanecieron menos de 3 meses en seguimiento. Se comparan el número de visitas al servicio de urgencias hospitalario, ingresos en planta y días de estancia en el año anterior a la atención por la unidad y durante el tiempo de seguimiento por la misma.Resultados: 81 pacientes con una edad media de 80,57 años (DE 7,39) y una mediana de atención por la unidad de 9 meses $(5-13,5)$, fueron incluidos en el estudio. Los consumos por paciente y mes de seguimiento disminuyeron en 0,07 visitas a Urgencias $(0,02-0,11)(\mathrm{p}=0,04), 0,10$ ingresos hospitalarios $(0,07-0,14)(\mathrm{p}<0,001)$, y 2,01 días de ingreso $(1,87-2,15)$ $(\mathrm{p}<0,001)$.

Conclusiones: Una unidad especializada de atención geriátrica domiciliaria disminuye el consumo de recursos hospitalarios de población anciana con enfermedad cardiorrespiratoria crónica.

Palabras clave: Atención sanitaria domiciliaria. Ancianos. Enfermedades crónicas. Enfermedad del sistema circulatorio. Enfermedad del sistema respiratorio.

Correspondencia:

Alejandro Pérez Martín

Unidad de Atención Domiciliaria

Hospital Geriátrico Virgen del Valle

Carretera Cobisa, $\mathrm{s} / \mathrm{n}$

45071 Toledo

Correo electrónico:aperezm@cht.insalud.es

\section{ABSTRACT}

The Effect on the Use of Hospital Resources in a Geriatric Home Care Program Among Elderly Advanced CardioRespiratory Disease Patients

Background: One of the objectives of the geriatric home care teams is that of the follow-up of elderly patients having a high risk of hospital readmission. Although they have been operating in our country for years, no data shows the impact on the use of hospital resources in accordance with this follow-up. The objective of this study is that of analyzing the effect which the monitoring, by geriatric home care unit, involves on patients having very advanced chronic cardiorespiratory disease.

Methods: The patients with chronic cardiorespiratory disease followed up by the unit during the January 1995-January 1999 period were included, excluding those on follow-up for less than 3 months. A comparison is drawn among the number of hospital emergency room visits, hospital admissions and days of hospitalization for the year prior to the care provided by the unit and throughout the follow-up time thereof are compared.

Results: Eighty-one (81) patients, mean age 80.57 years (DE 7.39) and a median length of care per unit of nine (9) months $(5-13,5)$, were included in the study. The uses per patient and month of follow-up decreased by 0.07 Emergency Room visits $(0.02-0.11)(\mathrm{p}=0.04), 0.10$ hospital admissions $(0.07-0.14)(\mathrm{p}<0.001)$ and 2.01 days of hospitalization $(1.87-2.15)(\mathrm{p}<0.001)$.

Conclusions: A specialized geriatric home care unit reduces the use of hospital resources on elderly people diagnosed as severe chronic cardiorespiratory disease.

Key words: Home Care Hospital-based, Aged. Chronic Disease. Cardiovascular disease. Respiratory tract disease. 


\section{INTRODUCCIÓN}

Los pacientes con enfermedades cardiorrespiratorias crónicas presentan episodios repetidos de reagudización que provocan gran número de visitas a los servicios de urgencias. Esta patología crónica suele encontrarse entre las causas más frecuentes de ingreso, tanto en nuestro país ${ }^{1}$ como en otros países desarrollados ${ }^{2-3}$.

Los incentivos del sistema de salud tienden a disminuir la estancia hospitalaria, lo que unido al progresivo envejecimiento poblacional y la aceptación por parte de los usuarios de sistemas de la atención domiciliaria ha originado un notable incremento de estos servicios ${ }^{4}$. Entre los principales procesos atendidos por estas unidades se encuentran las enfermedades cardiorrespiratorias crónicas. Una de las ventajas esperables del traslado de la atención al domicilio es un descenso en los días de hospitalización. Un reciente metaanálisis parece confirmar este resultado en diferentes programas de atención domiciliaria ${ }^{5}$. En él se destaca que la reducción de días de hospital varía en función del tipo de estudio, tipo de enfermo, tiempo de permanencia en programa, etcétera. En general parece ser que el efecto es mayor en enfermos terminales frente a los no terminales. Igualmente parece que cuanto más tiempo se mantienen en programa más difícil es conseguir el objetivo de disminuir el número de estancias hospitalarias.

En España existen intervenciones que analizan el efecto de los programas de hospitalización a domicilio sobre el consumo hospitalario de pacientes bronquíticos $\mathrm{y} / \mathrm{o}$ cardiópatas que demuestran un descenso en la utilización del hospital a corto plazo ${ }^{6-8}$.

Los equipos de atención geriátrica domiciliaria de base hospitalaria pretenden trasladar las características de la atención especializada al domicilio del anciano enfermo ${ }^{9}$. Intentan mediante un exhaustivo control clínico incrementar la permanencia del anciano en el domicilio sin mermar la calidad asistencial ${ }^{10}$. Su objetivo no se centra tanto a corto plazo sino en la selección de candidatos con alto riesgo de reingreso y el seguimiento más a largo plazo. Aunque llevan años funcionando en nuestro país no se tienen datos sobre el efecto que estos equipos representan sobre los consumos hospitalarios.

El presente trabajo tiene como objetivo analizar los cambios acaecidos en los consumos hospitalarios de pacientes con enfermedades cardiorrespiratorias crónicas que permanecen de forma regular en control por un equipo de atención geriátrica domiciliaria de base hospitalaria.

\section{SUJETOS Y MÉTODOS}

Se diseñó un estudio observacional retrospectivo de cohortes con controles históricos apareados donde cada sujeto es su propio control. El ámbito del estudio es el área de asistencia de la Unidad de Atención Geriátrica Domiciliaria del Hospital Virgen del Valle, que se encuentra circunscrita a una zona geográfica de unos 35 kilómetros alrededor de la ciudad de Toledo. Los sujetos de este estudio son personas con procesos cardiorrespiratorios crónicos que fueron seguidas por dicha unidad entre el 1 de enero de 1995 y el 15 de enero de 1999. En este período se evaluó a un total de 244 pacientes por patología cardiorrespiratoria crónica. De ellos, 140 $(57,4 \%)$ fueron incluidos en programa de atención domiciliaria. De los restantes 59 $(24,2 \%)$, fueron remitidos para seguimiento por su equipo de atención primaria $\mathrm{y}$ $43(17,6 \%)$ se consideró que debían ser atendidos por otro nivel asistencial (consulta externa hospitalaria, ingreso en unidad de agudos, etcétera.). De 2 casos no incluidos en el programa no se dispone de información sobre su atención. Dado que el objetivo del estudio es valorar el efecto de la intervención a largo plazo, se excluyó a los pacientes con tiempo de seguimiento breve; de esta forma además se limita la posible influencia estacional sobre la situación clínica de estos pa- 
cientes. La muestra del estudio la componen 81 pacientes que permanecieron al menos 3 meses en seguimiento por la unidad.

La variable de resultado del presente estudio es el consumo de recursos hospitalarios. Dicho consumo se evaluó en forma de tasa de visitas a urgencias, número de ingresos y días de estancia por paciente y mes de seguimiento. Para ello se calculó el numerador de dichas tasas como el número de visitas al servicio de urgencias hospitalario, número de ingresos en planta y número de días de estancia respectivamente. El denominador se compone de los meses analizados según el período de análisis que se considere. El período preintervención se consideró el año previo al seguimiento del enfermo por la unidad, por lo que representa una cifra fija de 12 meses por paciente. En el caso del período de intervención se consideran los meses que permanece en control por la unidad, bien hasta su alta de la misma o hasta la fecha de finalización del estudio. En el caso que el paciente permaneciera en control por tiempo superior al año, el tiempo de análisis de intervención se limita a los 12 meses posteriores a su inclusión en programa para igualar el tiempo de análisis al período preintervención.

El origen de los datos es el CMBD de los hospitales del Complejo Hospitalario de Toledo (área asistencial de la unidad) para el número de ingresos y los días de estancia, y el registro del servicio de admisión de urgencias hospitalarias para el número de visitas a dicho servicio.

Las variables independientes se captaron de la historia clínica estructurada que se realiza en el momento de la evaluación inicial (fecha de inicio de la intervención). En dicha historia se recogen de forma sistemática y protocolizada, junto a los elementos habituales de la misma, la presencia o ausencia de problemas geriátricos activos (caídas, incontinencia urinaria, etc.), valoración funcional, tanto de movilidad como de actividades básicas de autocuidado y presencia o ausencia de deterioro cognitivo y/o trastorno del ánimo. Igualmente se refleja el resultado de la evaluación y en caso que se decida incluirlo en programa de atención domiciliaria cuál es el motivo de dicha atención.

Los criterios que se siguen para evaluar estas variables se describen a continuación. La situación funcional se juzga mediante los parámetros de independencia en actividades básicas de autocuidado, capacidad de desplazarse fuera del domicilio y de movilización dentro del mismo. Se estima que un paciente presentaba un grado de dependencia leve en actividades de autocuidado, si requiere ayuda en menos de 2 actividades básicas de autocuidado (aseo, baño, vestido, trasferencia, ir al servicio, alimentación y continencia esfinteriana), dependencia moderada si requiere ayuda entre 2 y 5 actividades y dependencia severa si presenta dependencia en más de cinco actividades. Se considera que el enfermo se encuentra limitado al domicilio si es incapaz de deambular de forma autónoma fuera de su hogar (pueden utilizarse medios ortopédicos - bastón, andador, etc.- - ), y que se encuentra inmóvil si requiere ayuda de una persona o más para deambular dentro del hogar. La presencia de deterioro cognitivo se valora mediante la existencia de antecedentes de síndrome de demencia o trastorno de memoria que limita las capacidades habituales del paciente.

Por la posible repercusión sobre los consumos de recursos hospitalarios se clasificaron los pacientes como residentes en la ciudad de Toledo o en área rural si su residencia era cualquiera de las restantes poblaciones que cubre la unidad. La disponibilidad de cuidador se evaluó en función de si el paciente vivía solo, permanecía con un cuidador estable o bien variaba de cuidador con el tiempo.

Cuando un enfermo se consideraba candidato a ser incluido en el programa de atención domiciliaria el equipo que realizaba la valoración clasificaba la causa principal de atención por la unidad. Los motivos para ser 
atendidos por la unidad podían ser paliativos, control crónico cuando se apreciaba que el paciente presentaba patología crónica inestable de difícil control por su equipo de atención primaria y disminuir estancias hospitalarias cuando el número de consumos hospitalarios previos no se ajustaba a la situación clínica del paciente.

Descripción de la intervención: El tipo de intervención que recibieron los pacientes fue su inclusión para seguimiento por la unidad de atención geriátrica domiciliaria. El equipo de atención geriátrica domiciliaria es un equipo interdisciplinar compuesto por médicos, enfermeras y un trabajador social, cuyo objetivo es proveer atención sanitaria especializada en el domicilio de pacientes, habitualmente ancianos frágiles, que se encuadran en tres tipologías:

a. Pacientes terminales que precisan cuidados paliativos, oncológicos y no oncológicos.

b. Patología cardiaca y/o respiratoria en situaciones clínicas muy evolucionadas

c. Patología neurológica (accidentes cerebrovasculares con gran dependencia residual y demencias en estadios avanzados) con frecuentes complicaciones y/o difícil control sintomático.

Los criterios que se siguen para incluir un paciente con enfermedad cardiorrespiratoria crónica en el programa son de diversa índole:

1. Características clínicas: Enfermedad cardiorrespiratoria crónica muy evolucionada manifestada por disnea incapacitante (mínimos esfuerzos, reposo), requerimiento de oxigenoterapia domiciliaria, presencia de cor pulmonale y/o fracción de eyección inferior a 30\%. Situación inestable al alta hospitalaria con elevada sospecha de reingreso.

2. Relación previa con la atención hospitalaria: Presencia previa de 3 o más in- gresos y/o visitas al servicio de urgencias hospitalario en el último año.

3. Características de anciano frágil: limitados al domicilio por su enfermedad de base, dependencia en actividades de autocuidado, polifarmacia, etc.

4. Características sociales: Existencia de cuidador principal identificable.

Estos criterios son orientativos, pudiendo cumplirse tan solo uno o varios de ellos. En última instancia la inclusión de un paciente depende de la decisión del equipo que realiza la evaluación.

La actividad de la unidad con estos pacientes se realiza habitualmente de forma programada no prestándose atención urgente. El trabajo se organiza tras una valoración inicial conjunta por médico y enfermera que decide un plan de cuidados. En caso de permanecer el paciente estable es vigilado por la enfermera. En caso de reagudización o problema nuevo se revalúa al enfermo por el médico y la enfermera adaptando el plan de cuidados a la nueva situación. El seguimiento del paciente puede ser diario, semanal, quincenal o mensual de acuerdo a su situación clínica.

Análisis estadístico: La influencia del programa en los consumos de recursos hospitalarios se evaluó mediante las diferencias de tasas entre el período preintervención y el de intervención, calculándose para un intervalo de confianza al $95 \%$. Se utilizó el test de los rangos de Wilcoxon para muestras pareadas para comprobar si existían diferencias entre los consumos objetivados en ambos períodos. Se empleó el paquete estadístico SPSS v6.01 y EPI-INFO 6.04 para la realización de dichos cálculos.

\section{RESULTADOS}

En el período de estudio, 81 pacientes fueron seguidos durante tres o más meses por la unidad de atención domiciliaria. La edad media fue de 80,57 años (DE 7,39). Entre sus antecedentes patológicos destaca- 
ban: cardiopatías $63,75 \%$, hipertensión arterial $48,75 \%$, broncopatía crónica $41,25 \%$, diabetes mellitus $30 \%$ y fibrilación auricular $23,75 \%$. En el momento de su evaluación inicial tomaban un promedio de 7,5 fármacos. En la tabla 1 se presentan las características demográficas, origen de los pacientes y situación funcional.

Tabla 1

Características de los sujetos incluidos en el estudio

\begin{tabular}{|c|c|c|c|}
\hline & $\begin{array}{l}\text { Número pacientes } \\
\text { incluidos en el } \\
\text { programa }\end{array}$ & 81 & $\%$ \\
\hline \multirow[t]{3}{*}{ Origen } & $\begin{array}{l}\text { Equipo atención } \\
\text { primaria }\end{array}$ & 3 & 3,8 \\
\hline & Hospital & 68 & 85,0 \\
\hline & Urgencias & 9 & 11,3 \\
\hline \multirow[t]{3}{*}{ Edad } & $65-74$ & 17 & 22,4 \\
\hline & $75-84$ & 36 & 47,4 \\
\hline & 85 y más & 23 & 30,3 \\
\hline \multirow[t]{2}{*}{ Sexo } & Varón & 39 & 48,1 \\
\hline & Mujer & 42 & 51,9 \\
\hline \multirow[t]{2}{*}{ Residencia } & Toledo & 16 & 19,8 \\
\hline & Resto & 65 & 80,2 \\
\hline \multirow[t]{3}{*}{ Estado civil } & Casado/a & 45 & 56,3 \\
\hline & Viudo/a & 33 & 41,3 \\
\hline & Soltero/a & 2 & 2,5 \\
\hline \multirow{3}{*}{$\begin{array}{l}\text { Dependencia } \\
\text { autocuidado }\end{array}$} & Leve & 15 & $18,8 \%$ \\
\hline & Moderada & 26 & $32,5 \%$ \\
\hline & Severa & 39 & $48,8 \%$ \\
\hline \multirow[t]{2}{*}{ Inmovilidad } & no & 61 & $76,3 \%$ \\
\hline & si & 19 & $23,8 \%$ \\
\hline \multirow[t]{2}{*}{ Limitación domicilio } & No & 14 & $17,5 \%$ \\
\hline & $\mathrm{Si}$ & 66 & $82,5 \%$ \\
\hline \multirow{2}{*}{ Deterioro cognitivo } & No & 70 & $90,9 \%$ \\
\hline & Sí & 7 & $9,1 \%$ \\
\hline \multirow{3}{*}{$\begin{array}{l}\text { Presencia de } \\
\text { cuidador }\end{array}$} & Solo & 0 & $0,0 \%$ \\
\hline & Estable & 63 & $87,5 \%$ \\
\hline & Itinerante & 9 & $12,5 \%$ \\
\hline \multirow[t]{3}{*}{ Motivo de ingreso } & Paliativo & 1 & $1,2 \%$ \\
\hline & Control crónico & 48 & $59,3 \%$ \\
\hline & Disminuir estancias & 32 & $39,5 \%$ \\
\hline
\end{tabular}

La discrepancia con el total de pacientes (81) corresponde a datos perdidos
Los pacientes permanecieron una mediana de 9 meses (rango intercuartílico $5-13,50)$ en atención por la unidad. Se verificó que 22 pacientes $(27,2 \%)$, permanecieron más de 12 meses en seguimiento por la unidad, por este motivo el tiempo mediano de intervención analizado fue de 9 meses (rango intercuartílico 5-12). En el período de intervención los pacientes recibieron una mediana de 14 (rango intercuartílico 11-23) visitas del equipo de enfermería y 4 (rango intercuartílico 2-8) visitas del equipo médico. Un total de 22 pacientes $(27,2 \%)$, fueron dados de alta por estabilización prolongada de su cuadro clínico y en 12 casos $(14,8 \%)$ se mantenía el seguimiento en el momento de finalizar el estudio. La causa más habitual de alta de la unidad fue el fallecimiento, 47 pacientes (58\%), que tuvo lugar en el domicilio del enfermo en 38 casos $(80,9 \%)$.

En los 972 meses de período preintervención se contabilizaron 239 visitas a urgencias y 173 ingresos hospitalarios con un total de 2.805 días de estancia. En 696 meses de seguimiento de intervención se objetivaron 125 visitas a urgencias y 52 ingresos, con un total de 611 días de estancia. En la tabla 2 se reflejan las tasas por paciente y mes de seguimiento que presentaban los antes y durante su atención por la unidad. Se produce un descenso en términos relativos del $28 \%$ del número de visitas a urgencias, del 55,55\% del número de ingresos, y del 69,55\% del número de días de estancia.

La influencia de la covariables analizadas en el descenso de consumos se presenta en la tabla 3. No se objetivaron diferencias significativas en ninguna de las variables analizadas con respecto al global.

El descenso de consumo de recursos hospitalarios por paciente y año de seguimiento se resumen en $0,84(0,24-1,32)$ visitas al servicio de urgencias, $1,20(0,84-1,68)$ ingresos hospitalarios y $24,12(22,44-25,80)$ días de estancia. 
Tabla 2

Consumo de recursos hospitalarios por paciente y mes de seguimiento

\begin{tabular}{|lrcrcrc|}
\hline \multicolumn{4}{c}{ Preintervención } & \multicolumn{2}{c|}{ Postintervención } \\
\cline { 2 - 7 } & \multicolumn{2}{c}{$\begin{array}{c}\text { Eventos/ } \\
\text { pac.mes }\end{array}$} & Tasa & $\begin{array}{c}\text { Eventos/ } \\
\text { pac.mes }\end{array}$ & Tasa & $\begin{array}{c}\text { Diferencia } \\
\text { tasas } p\end{array}$ \\
\hline Urgencias & $239 / 972$ & $0,25(0,19,0,30)$ & $125 / 696$ & $0,18(0,14,0,24)$ & $0,07(0,02,0,11)$ & 0,04 \\
Ingresos & $173 / 972$ & $0,18(0,15,0,21)$ & $52 / 696$ & $0,08(0,05,0,11)$ & $0,10(0,07,0,14)$ & $<0,001$ \\
Días estancia & $2805 / 972$ & $2,89(2,39,3,39)$ & $611 / 696$ & $0,88(0,55,1,32)$ & $2,01(1,87,2,15)$ & $<0,001$ \\
\hline
\end{tabular}

Consumos hospitalarios por paciente y mes de seguimiento en los 12 meses previos (Preintervención) y después de su inclusión en la Unidad de Atención Geriátrica Domiciliaria (Intervención).

Se ofrecen las tasas con su intervalo de confianza al 95\%. Eventos/pac.mes: Número de eventos por paciente y mes de seguimiento.

Tabla 3

Diferencias de riesgo en los períodos preintervención y de intervención evaluadas mediante diferencia de tasas por paciente y mes de seguimiento en función de sus características

\begin{tabular}{|c|c|c|c|c|}
\hline & & Urgencias & Ingresos & Estancias \\
\hline \multirow[t]{3}{*}{ Edad } & $65-74$ & $0,07(-0,04,0,19)$ & $0,12(0,02,0,22)$ & $2,42(2,05,2,79)$ \\
\hline & $75-84$ & $0,04(-0,03,0,11)$ & $0,96(0,45,1,47)$ & $1,91(1,69,2,11)$ \\
\hline & 85 y más & $0,10(0,03,0,18)$ & $0,11(0,05,0,16)$ & $1,96(1,75,2,17)$ \\
\hline \multirow[t]{2}{*}{ Sexo } & Varón & $0,05(-0,02,0,12)$ & $0,11(0,05,0,16)$ & $2,08(1,87,2,28)$ \\
\hline & Mujer & $0,08(0,02,0,14)$ & $0,10(0,05,0,14)$ & $1,94(1,78,2,13)$ \\
\hline \multirow[t]{2}{*}{ Localidad } & Toledo & $0,08(0,03,0,13)$ & $0,11(0,07,0,15)$ & $1,92(1,77,2,08)$ \\
\hline & Resto & $0,03(-0,08,0,13)$ & $0,14(0,05,0,24)$ & $2,42(2,07,2,77)$ \\
\hline \multirow[t]{3}{*}{ Estado civil } & Casado & $0,03(-0,04,0,09)$ & $0,10(0,04,0,15)$ & $1,84(1,64,2,03)$ \\
\hline & Soltero & $0,26(-0,12,0,65)$ & $0,17(-0,12,0,47)$ & $2,13(1,08,3,17)$ \\
\hline & Viudo & $0,15(0,04,0,17)$ & $0,10(0,05,0,15)$ & $2,10(1,90,2,29)$ \\
\hline \multirow[t]{3}{*}{ DEPENDENCIA ABVD } & Leve & $0,09(-0,11,0,29)$ & $0,07(-0,06,0,20)$ & $1,15(0,69,1,61)$ \\
\hline & Moderada & $0,03(-0,08,0,13)$ & $0,09(0,01,0,18)$ & $2,14(1,81,2,47)$ \\
\hline & Severa & $0,10(0,03,0,18)$ & $0,12(0,06,0,19)$ & $1,61(1,37,1,84)$ \\
\hline \multirow[t]{2}{*}{ Limitado domicilio } & No & $0,05(-0,00,0,12)$ & $0,10(0,05,0,15)$ & $2,34(2,13,2,55)$ \\
\hline & Sí & $0,08(0,02,0,15)$ & $0,10(0,05,0,16)$ & $1,67(1,48,1,86)$ \\
\hline \multirow[t]{2}{*}{ Deterioro cognitivo } & No & $0,05(0,01,0,10)$ & $0,10(0,06,0,14)$ & $2,02(1,88,2,16)$ \\
\hline & Sí & $0,38(0,09,0,66)$ & $0,16(-0,01,0,32)$ & $1,81(1,25,2,37)$ \\
\hline \multirow[t]{3}{*}{ Motivo alta } & Fallecimiento & $0,10(0,04,0,17)$ & $0,11(0,06,0,15)$ & $2,15(1,96,2,35)$ \\
\hline & Estabilización & $0,04(-0,05,0,13)$ & $0,09(0,02,0,17)$ & $1,90(1,62,2,18)$ \\
\hline & En programa & $-0,00(-0,12,0,17)$ & $0,11(0,03,0,18)$ & $1,53(1,26,1,79)$ \\
\hline Global & & $0,07(0,02,0,11)$ & $0,10(0,07,0,14)$ & $2,01(1,87,2,15)$ \\
\hline
\end{tabular}

Los números positivos corresponden a descensos en el consumo mientras que los negativos representan incrementos de consumos en el período de intervención. Dependencia ABVD (dependencia en actividades de autocuidado). 


\section{DISCUSIÓN}

Nuestros resultados presentan el efecto de una unidad de atención geriátrica domiciliaria de base hospitalaria en el manejo de pacientes cardiorrespiratorios crónicos muy evolucionados. Del estudio se desprende que es posible modificar los consumos hospitalarios que realizan los pacientes ancianos de alto riesgo mediante intervenciones especializadas en su tratamiento.

El programa de atención geriátrica domiciliaria disminuye de forma notable el consumo de recursos hospitalarios. Sin embargo el descenso tanto del número de días de estancia como del número de ingresos, aunque superan el $50 \%$ de reducción, se encuentran alejados de los publicados en nuestro país. Una intervención de base hospitalaria sobre enfermos con patología respiratoria crónica describe una disminución del $75 \%$ en el consumo de estancias 7 . Un programa de hospitalización domiciliaria sobre mayores de 70 años fue capaz de reducir por encima del $86 \%$ los ingresos de hospitalización convencional en caso de insuficiencia cardíaca. Ambas intervenciones demuestran una reducción de consumos superior a la alcanzada en nuestra unidad. Las diferencias se pueden deber a: 1) actuación sobre población más joven, 2) período de tiempo analizado preintervención breve en el caso de la unidad de hospitalización domiciliaria (90 días $)^{6}$ y 3 ) seguimientos limitados en el tiempo. Habitualmente la derivación de enfermos a estas unidades se realiza tras un ingreso hospitalario o visita al servicio de urgencias, por lo que el consumo previo, si se centra en un período breve de análisis, puede encontrarse sobrestimado. Por otra parte, el seguimiento prolongado en el tiempo parece disminuir la eficacia con respecto al consumo de días de hospitalización ${ }^{5}$. Estos hechos junto a la existencia de cierta tendencia estacional en las reagudizaciones de estas patologías nos han llevado a seleccionar a los pacientes con más de 3 meses de seguimiento y analizar los consumos realizados en el año previo.
En la literatura internacional intervenciones domiciliarias de origen en el hospital que actúan sobre patología de forma específica (respiratoria o cardiológica) muestran descensos en los consumos de días de hospital que oscilan entre el 40 y $49 \%{ }^{11-13}$. Sobre población geriátrica no seleccionada por patología, el descenso oscila entre el $30 \%{ }^{14}$ y $55 \%{ }^{15}$. Creemos que la intervención descrita por Cummings es la que más se asemeja a nuestra unidad, tanto por población sobre la que actúa como por los resultados obtenidos. En ella se objetiva un claro descenso en el consumo de días de hospital en torno al $30 \%$, mientras que el número de visitas a urgencias, al igual que ocurre en nuestro estudio, se reduce de forma notablemente inferior, en torno al $17 \%$ frente al $24 \%$ en nuestro caso. Por el tipo de patología atendida no es de sorprender que se produzcan frecuentes reagudizaciones en horario no cubierto por la unidad y que ello origine el desplazamiento al servicio de urgencias, y que sea éste, por ende, el consumo que menos se puede influir por la existencia de atención domiciliaria como la aquí descrita.

Existe una alta mortalidad, $58 \%$, en el grupo atendido. Esta cifra es superior a las que se presentan en otros estudios, que la sitúan entre el 12 y el 30\% ${ }^{11-15}$. Estas cifras de mortalidad se pueden explicar, además de por la edad y patología de base, por la situación funcional, que es un elemento fundamental a tener en cuenta a la hora de emitir el pronóstico de un paciente ${ }^{16}$. Los pacientes del presente estudio, por encima del $80 \%$ limitados al domicilio e inmóviles en el $23 \%$, con una tasa de dependencia moderada-severa en actividades de autocuidado superior en el $80 \%$ de los pacientes, son probablemente las variables que mejor explican la alta mortalidad durante su seguimiento.

La edad, patología de base y características funcionales de las personas de nuestra muestra se corresponde con la de los pacientes que mayor riesgo de ingreso hospitalario presentan ${ }^{17}$. La reducción de los consumos puede deberse en parte a que la muestra no 
es homogénea en sus períodos de comparación. La evolución de la enfermedad, con su tendencia a la progresión, puede hacer que se considere el hospital como poco útil en la atención de estos enfermos. Sin embargo, la mayor parte de las estancias hospitalarias de los ancianos tienen lugar en el último año de vida, con múltiples reingresos, siendo mucho más frecuente que fallezcan en el hospital ${ }^{18,19}$. Por lo tanto, en nuestro caso puede esperarse de forma razonable que la evolución del cuadro hubiese dificultado conseguir una reducción de las estancias. Es de destacar que sobre esta población con alto consumo de recursos hospitalarios se pueda producir un ahorro por enfermo y año de permanencia en la intervención que puede oscilar entre 22 y 26 días de ingreso hospitalario, y que la misma se realice en el último año de vida en la mayoría de los casos.

Entre las limitaciones de este trabajo están el que se trata de un estudio retrospectivo con escaso número de sujetos. La forma de trabajo de la Unidad con datos recogidos de forma protocolizada ayuda a soslayar este problema. De hecho, el carácter retrospectivo puede considerarse una ventaja a la hora de evitar el efecto hawthorne (respuesta inducida por conocimiento de ser observado). Comprobamos el efecto del programa bajo funcionamiento real, sin presiones que alteren la dinámica habitual. Aunque sería deseable un estudio randomizado para evitar sesgos y disponer de un grupo control, este tipo de intervenciones sobre población como la aquí expuesta resulta muy difícil que sean ciegas y que no presenten un efecto paralelo sobre el grupo control ${ }^{20}$. Junto a ello es probable que la actitud de los miembros del equipo forzara situaciones clínicas y sociales complejas. Por este motivo este tipo de estudio "antes-después», conociendo sus limitaciones, nos parece una aproximación razonable al problema. Conjuntamente la dimensión de la reducción de los días de estancia parece de suficiente entidad como para poder valorar el efecto del programa de atención domiciliaria.
Aunque la unidad de atención domiciliaria es un recurso que habría que incluir en los consumos hospitalarios, su coste es muy inferior al de los días de estancia. Además, probablemente represente una modificación del consumo de otros recursos no analizados (consultas al equipo de atención primaria, consultas especializadas, desplazamientos, etcétera). El carácter retrospectivo del estudio impide realizar un análisis de coste-efectividad mínimamente riguroso.

La selección de enfermos atendida puede hacer que los resultados de la misma no sean reproducibles. Aunque pueda ser una crítica, la realización de cualquier intervención sobre población geriátrica requiere seleccionar aquellos candidatos más idóneos para la misma. Parece evidente que la reducción de las visitas a urgencias, de los ingresos y de los días de estancia se realiza a expensas de un número importante de visitas médicas y de enfermería. Por este motivo se debe centrar la actividad sobre ancianos de alto riesgo y con elevado coste sanitario para que el resultado de la atención domiciliaria puede ser eficiente ${ }^{21}$.

\section{AGRADECIMIENTOS}

A los Drs. A Carbonell Collar y FJ García García por su desinterada revisión y comentarios realizados sobre el presente manuscrito.

\section{BIBLIOGRAFÍA}

1. González Montalvo JI, Bastan JJ, Rodríguez Mañas L, San Cristobal E, Gato A, Ballesteros P, et al. Ingreso hospitalario urgente en servicios médicos: causas, justificación, factores sociales y sus diferencias en función de la edad. Med Clin (Barc) 1994;103:441-4.

2. Blaum CS, Liang J, Liu X. The relationship of chronic diseases and health status to health services uitlization of older americans. J Am Geriatr Soc 1994;42:1087-93.

Rev Esp Salud Pública 2001, Vol. 75, N. ${ }^{\circ} 6$ 
3. Cusker JM, Healey E, Bellavance F, Connolly B. Predictors of repeat emergency department visits by elders. Acad Emerg Med 1997;4:581-8.

4. Burton LC, Leff B, Harper M, Ghoshtagore I, Steinwachs DA, Greenough WB, et al. Acceptability to patients of a home hospital. J Am Geriatr Soc 1998;46:605-9.

5. Hughes SL, Ulasevich A, Weaver FM, Henderson W, Manheim L, Kubal JD et al. Impact of home care on hospital days: A metaanalaysis. Health Serv Res 1997;32:415-32.

6. Bechich S, Sort Granja D, Arroyo Mateo X, Delás Amat J, Rosell Abaurrea F. Efecto de la hospitalización a domicilio en la reducción de la hospitalización convencional y frecuentación de urgencias en la insuficiencia cardíaca. Rev Clin Esp 2000;200:310-14.

7. Güell R, González A, Morante F, Sanguenis M, Sotomoayor C, Caballero C, et al. Mejor en casa: un programa de asistencia continuada para los pacientes con enfermedad respiratoria crónica avanzada. Arch Bronconeumol 1998;34:541-6.

8. Farrero E, Escarrabill J, Prats E, Maderal M, Manresa F. Impact of a hospital-based home-care program on the management of COPD patients receiving long-term oxygen therapy. Chest 2001;119:364-9.

9. Serra Rexach JA, Rexach Cano L, Cruz Jentoft AJ, Gil Gregorio P, Ribera Casado JM. Asistencia geriátrica domiciliaria: veinte meses de experiencia. Rev Clin Esp 1992;191:405-11.

10. González Montalvo JI, Pérez del Molino J, Rodríguez Mañas L, Salgado Alba A, Guillén Llera F. Geriatría y asistencia geriátrica: Para quién y cómo (I). Med Clin (Barc) 1991;96:183-8.

11. Haggerty MC, Stockdale-Woolley R, Nair S Respi-Care. An innovative home care program for the patient with chronic obstructive pulmonary disease. Chest 1991;100:607-12.

12. Stewart S, Vanderbrock AJ, Pearson S, Horowitz JD. Prolonged beneficial effects of a home-based intervention on unplanned readmissions and mortality among patients with congestive heart failure. Arch Intern Med 1999; 159:257-61.

13. Rich MW, Beckham V, Wittemberg C, Leven CL, Freedland KE, Carney RM. A multidisciplinary intervention to prevent the readmission of elderly patients with congestive heart failure. $\mathrm{N}$ Engl J Med 1995;333:1184-9.

14. Cummings JE, Hughes SL, Weaver FM, Manheim LM, Conrad KD, Nash K et al. Cost-effectiveness of veterans administration hospital-based home care. A randomized clinical trial. Arch Intern Med 1990;150:1274-80.

15. Landi F, Gambassi G, Pola R, Tabaccanti S, Cavinato T, Carbonin P, Bernabei R. Impact of integrated home care services on hospital use. J Am Geriatr Soc 1999;47:1430-4.

16. Reuben DB, Rubenstein LV, Hirsch SH, Hays RD. Value of functional status as a predictor of mortality: results of a prospective study. Am J Med 1992;93:663-9.

17. Pérez Martín A, García García FJ, Martín Correa E, Romero Rizos L, Rodríguez Barquero MJ, Echevarría Santamaría I, et al. Factores de riesgo de hospitalización en mayores de 64 años. Gac Sanit 2000:14:363-370.

18. Burns R, Nichols LO. Factors predicting readmission of older general medicine patients. J Gen Intern Med 1991;6:389-393

19. Roos NP, Roos LL, Mossey J, Havens B. Using administrative database to predict important health outcomes: entry to hospital, nursing home and death. Med Care 1988; 26:221-239.

20. Grande GE, Todd CJ, Barclay SI, Farquhar MC. Does hospital at home for palliative care facilitate death at home? Randomised controlled trial. BMJ 1999;319:1472-5.

21. Baztán Cortés JJ, González-Montalvo JI, Solano Jaurrieta JJ, Hornillos Calvo M. Atención sanitaria al anciano frágil: de la teoría a la evidencia científica. Med Clin (Barc) 2000;115:704-717. 